\title{
Prospecciones en la confluencia de los cañadones Caracoles-Pinturas. Santa Cruz, Argentina
}

\section{Mariana Sacchi}

(D) https://orcid.org/0000-0003-4014-1580

Carrera de Arqueología, Universidad Católica de Temuco - Facultad de Filosofía y Letras, Universidad de Buenos Aires (UBA). 3 de febrero 1378 (CP C1426BJN) Ciudad Autónoma de Buenos Aires. E-mail: sacchi.mariana@gmail.com

\section{Damián Bozzuto}

\section{(iD) https://orcid.org/0000-0001-6272-1338}

Instituto Nacional de Antropología y Pensamiento Latinoamericano (INAPL) - Consejo Nacional de Investigaciones Científicas y Técnicas (CONICET). 3 de febrero 1378 (CP C1426BJN) Ciudad Autónoma de Buenos Aires. E-mail: damianbozzuto@gmail.com

\section{Valeria Ucedo}

Parque Provincial Cueva de las Manos. Municipalidad de Perito Moreno. Av. San Martín 1776 (CP 9040), Perito Moreno, Santa Cruz, Argentina. E-mail: valeriaucedo@gmail.com

\author{
Nicolás Maveroff \\ (iD https://orcid.org/0000-0002-1457-0472 \\ Instituto Nacional de Antropología y Pensamiento Latinoamericano (INAPL) - Consejo Nacional de Investigaciones \\ Científicas y Técnicas (CONICET). 3 de febrero 1378 (CP C1426BJN) Ciudad Autónoma de Buenos Aires. E-mail: \\ nicolasmaveroff@gmail.com
}

\section{Emilia Daldin}

Instituto Nacional de Antropología y Pensamiento Latinoamericano (INAPL). 3 de febrero 1378 (CP C1426BJN) Ciudad Autónoma de Buenos Aires. E-mail: meli.daldin@gmail.com

\section{Agustina Papú}

Instituto Nacional de Antropología y Pensamiento Latinoamericano (INAPL) - Consejo Nacional de Investigaciones Científicas y Técnicas (CONICET). 3 de febrero 1378 (CP C1426BJN) Ciudad Autónoma de Buenos Aires. E-mail: agus.papu@gmail.com

Recibido: 29 de marzo de 2020 Aceptado: 9 de septiembre de 2020

\begin{abstract}
Resumen
Se presentan los primeros resultados de los relevamientos realizados en la confluencia de los cañadones Caracoles y Pinturas, en el noroeste de la provincia de Santa Cruz. Entre las tareas realizadas se destaca la ubicación de los sitios identificados por Gradin y colaboradores, un primer registro del arte rupestre en uno de ellos y la realización de transectas dirigidas. El análisis preliminar del material lítico recuperado mostró la utilización de rocas silíceas de muy buena calidad y de obsidiana para la confección de raspadores y puntas de proyectil pedunculadas. Uno de los objetivos finales de estos trabajos es identificar la profundidad temporal en el uso de este espacio particular para evaluar el papel que pudo haber tenido en la circulación humana hacia los sectores cordilleranos. Si bien los sondeos realizados no brindaron evidencias que pudieran datarse, la presencia de fragmentos cerámicos los vincula con ocupaciones que se ubicarían en el Holoceno tardío.
\end{abstract}




\title{
Surveys at Caracoles-Pinturas canyons confluence. Santa Cruz, Argentina
}

\begin{abstract}
The initial results of surveys carried out at the confluence of Caracoles and Pinturas canyons, in the northwest of Santa Cruz province, are presented. The tasks carried out included locating the sites identified by Gradin and collaborators, the initial recording of the rock art in one of them, and surveying using directed transects. The preliminary analysis of the recovered lithic material showed the selection of high-quality siliceous rocks and obsidian for making scrapers and stemmed projectile points. One of the ultimate goals of our research is to identify the temporal depth in the use of this particular area to evaluate the role it could have had in the human circulation towards the mountain-range area. Although the surveys did not provide datable evidence, the assemblages recovered are associated with ceramic sherds related to occupations that can be placed during the Late Holocene.
\end{abstract}

KEYWORDS: Circulation; Chronology; Pinturas River Basin; Caracoles Canyon.

\section{Introducción}

En este trabajo presentamos los resultados de una serie de prospecciones realizadas en el cañadón Caracoles, provincia de Santa Cruz. Se trata de un acercamiento al área esteparia luego de varios años de investigación en las cuencas lacustres cordilleranas (Aschero et al., 2009, 2019a; Fernández et al., 2019; Horta, Marcos, Bozzuto, Mancini y Sacchi 2016; Horta et al., 2019; Sacchi et al., 2016; entre otros). Estas investigaciones se enmarcan en proyectos mayores que tienen como objetivo explorar las relaciones existentes entre las ocupaciones de los ambientes cordilleranos (cuenca del lago Pueyrredón-Cochrane y zona del Parque Nacional Perito Moreno, PNPM de aquí en adelante), las del área del río Pinturas, la altiplanicie sur (área de laguna de los Cisnes) y el borde oriental de la alta meseta del lago Buenos Aires (Figura 1A). Los vínculos mencionados fueron propuestos en primer lugar entre las áreas del río Pinturas y de las cuencas lacustres cordilleranas. Inicialmente estas relaciones se plantearon a partir de similitudes en las representaciones rupestres y las características generales de los artefactos líticos (Aschero, 1981-1982). Con el avance de las investigaciones se sumaron nuevos datos a esos primeros indicadores de vínculos entre las diferentes áreas. Entre estos se encuentran las dataciones tempranas, el uso de obsidiana procedente de Pampa del Asador desde las primeras ocupaciones y la similitud en los estilos más tempranos de las representaciones rupestres que se comparten, por ejemplo, entre Cueva de las Manos y Cerro Casa de Piedra en el PNPM (Civalero, 2016; Civalero y Aschero, 2003). Por otro lado, también se amplió el conocimiento acerca de las similitudes en las características tecnológicas de los materiales líticos entre el PNPM, Cueva Milodón Norte 1 (ubicada en el lago Pueyrredón) y Cueva de las Manos (Sacchi et al., 2016). En este sentido, los proyectos que enmarcan este trabajo buscan evaluar las relaciones a lo largo del Holoceno entre las diferentes áreas mencionadas con el fin de rastrear la movilidad y la posible existencia de territorios y/o circuitos de interacción social con cierta estabilidad temporal (Aschero et al., 2019a; Fernández et al., 2019; Horta et al., 2016, 2019; entre otros). Esta continuidad de las interacciones a lo largo del tiempo habría estado dada por las evidencias de los vínculos entre la zona de estepa y cañadones de Cueva de las Manos y la de las cuencas lacustres cordilleranas desde los momentos iniciales de ocupación, hace alrededor de 10.000 años. Es así que se torna relevante la identificación de posibles vías de comunicación entre una zona y otra. Una de estas potenciales vías de circulación desde el río Pinturas hacia el Oeste sería el recorrido de este cañadón hasta la zona de Bajo Caracoles. 


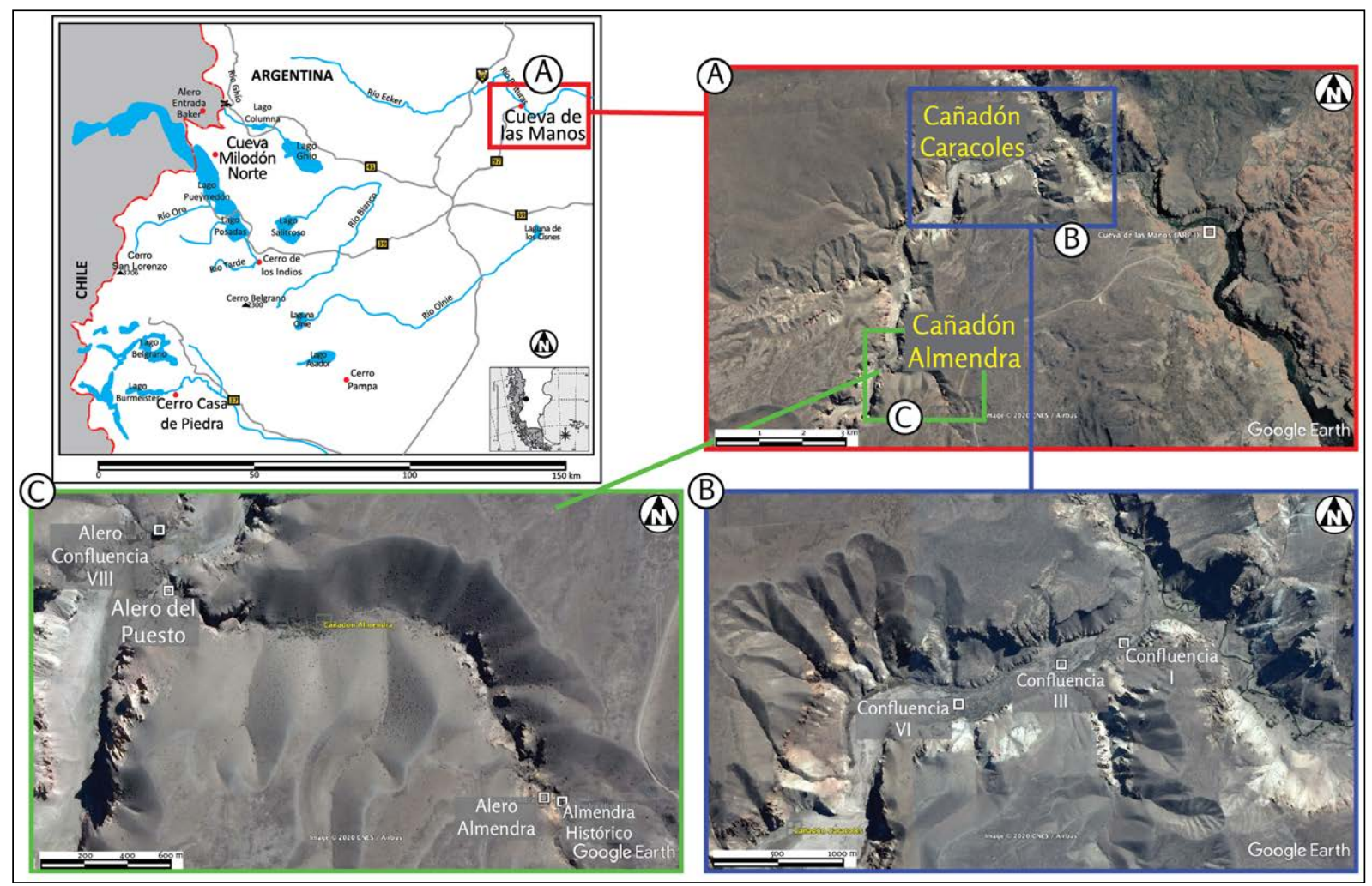

Figura 1. Vista general de las áreas estudiadas: A) vista general del área presentada; $B$ ) detalle del sector Confluencia Pinturas-Caracoles; $C$ ) detalle del cañadón Almendra.

El cañadón Caracoles es uno de los tributarios del río Pinturas, tiene unos $38 \mathrm{~km}$ de longitud y corre en sentido Suroeste-Noreste, desde la actual ruta nacional 40 hasta desembocar en el río Pinturas (Figura 1A). Como explican Geuna y Escoteguy (2008), el valle de este río, pese a que hoy solo drena las aguas provenientes de la meseta del lago Buenos Aires, se formó producto de los deshielos de los lagos Posadas y Pueyrredón. Según estos autores, el paisaje actual, caracterizado por ser una meseta esteparia con presencia de agua potable concentrada en los cañadones profundos, es producto de la acción de los ríos combinada con procesos de remoción en masa. Una de las características de este cañadón es que, en época de deshielo (entre octubre y diciembre), debido al desagote de diferentes vertientes se forman algunas lagunas. En estas pueden observarse flamencos (Phoenicopterus chilensis), cauquenes (Chloephaga sp.), choiques (Rhea pennata pennata) y guanacos (Lama guanicoe). En época estival estas lagunas se secan y se convierten en zonas de salitre, lo que dificulta el tránsito ya que por debajo de la superficie todavía concentran humedad. En cambio, durante otoño e invierno el cañadón permanece seco. Dadas sus características y su ubicación geográfica, puede ser entendido como una vía de circulación entre diferentes ambientes de la zona debido a que conecta la cuenca del Pinturas y el sector de los cañadones (en la estepa) con la actual ruta nacional 40 como forma de acceder a los sectores lacustres cordilleranos. De acuerdo con Musters (1911 [1871]), la zona que hoy es atravesada por la ruta nacional 40 era una importante vía de circulación Norte-Sur que a su vez constituye la puerta de acceso al bajo cordillerano donde se ubican los lagos Salitroso, Posadas y Pueyrredón-Cochrane (Aguerre, 1990-1992; Aschero et al., 2009; Belardi, 2004; Belardi, Tiveri, Stern y Súnico, 2006; Gradin y Aguerre, 1988; Horta et al., 2016; entre otros).

Por otra parte, en lo relacionado con la protección legal, el cañadón está incluido dentro de la Ley Provincial $N^{\circ} 3.394$ y el Decreto Nacional $N^{\circ} 817 / 2019$. Estas leyes establecen una protección para toda el área comprendida por la cuenca del río Pinturas resguardando no sólo los bienes arqueológicos sino también el 


\begin{tabular}{|l|c|c|c|c|}
\hline & Confluencia I & Confluencia III & Confluencia VI & Total \\
\hline Artefactos formatizados & 141 & 4 & - & 145 \\
Núcleos & 26 & 4 & - & 30 \\
Filos naturales con rastros complementarios & 10 & 6 & - & 16 \\
Desechos de talla & 1968 & 33 & - & 2001 \\
Fragmentos de vidrio & - & - & 25 \\
Tiestos & 13 & - & - & 13 \\
Machacadores & 1 & - & - & 1 \\
Cuentas de collar & - & 48 & 25 & 1 \\
\hline Total & 2159 & & 2232 \\
\hline
\end{tabular}

Tabla 1. Concentraciones identificadas y tipo de material recuperado.

paisaje, con una definición amplia del mismo. Si bien este sector específico no se encuentra dentro del área del Parque Provincial Cueva de las Manos (Ley Provincial $\left.\mathrm{N}^{\circ} 3.613 / 2018\right)$, sí está incluido dentro del área de influencia conformada en su plan de manejo (en desarrollo y próximo a ser presentado) para la preservación de la cuenca del río Pinturas, el Parque Nacional Patagonia y los propietarios privados vecinos del Parque.

\section{Antecedentes}

En lo que se refiere a la arqueología, el área del Alto Río Pinturas (ARP) ha sido intensivamente estudiada durante las décadas de 1970, 1980, 1990 y, recientemente, se han retomado las investigaciones, haciendo especial énfasis sobre el arte rupestre (Aguerre, 1990-1992, 2000, 2003; Aguerre y Gradin, 2003; Alonso, Gradin, Aschero y Aguerre, 1984-1985; Aschero, Isasmendi, Ucedo y Aguerre, 2019b; Gradin, Aschero y Aguerre, 1976, 1979, 1987; entre otros). Estas manifestaciones pictóricas ya habían sido mencionadas por el padre Agostini y Vignati en la primera mitad del siglo XX (Gradin et al., 1976).

Una de las primeras referencias al cañadón Caracoles -en términos arqueológicosfue hecha por Gradin et al. (1979) cuando identificaron una serie de sitios de superficie y aleros ubicados en cañadones adyacentes. Entre estos se destaca ARP III, ubicado en la confluencia entre los cañadones Caracoles y Pinturas. El sitio fue definido como un taller-campamento extendido, con abundantes artefactos en superficie, en el que se realizaron recolecciones en las campañas de 1973 y 1976. Ese material fue agrupado en el nivel Río Pinturas V, que presenta como rasgo característico artefactos de módulo pequeño, un creciente aumento de artefactos con retoque bifacial extendido y puntas pedunculadas pequeñas de limbo triangular (Gradin et al., 1979). En cuanto al cañadón como vía de acceso y comunicación entre la región lacustre cordillerana y la estepa, los autores comentan que el mismo Musters, durante su exploración del área, lo habría aprovechado para llegar hasta el río Pinturas y desde allí ascender hacia el sector alto muy cerca de la Estancia Sumich (Gradin et al., 1979). En esa zona se ubicaría la "colina de dios" mencionada por Musters, que más tarde Escalada (1949) señalaría como posible lugar de origen de los hombres y animales según la cosmogonía tehuelche (Gradin et al., 1979).

El Caracoles cuenta con varios cañadones transversales, en los cuales Gradin y colaboradores (1979) identificaron un alero ubicado en el curso alto del cañadón Almendra. Este alero, denominado "Almendra" (ARP XI en Gradin et al., 1979) presenta evidencias de negativos de mano y artefactos líticos en el talud (Figura $1 \mathrm{C}$ y Figura 2). 


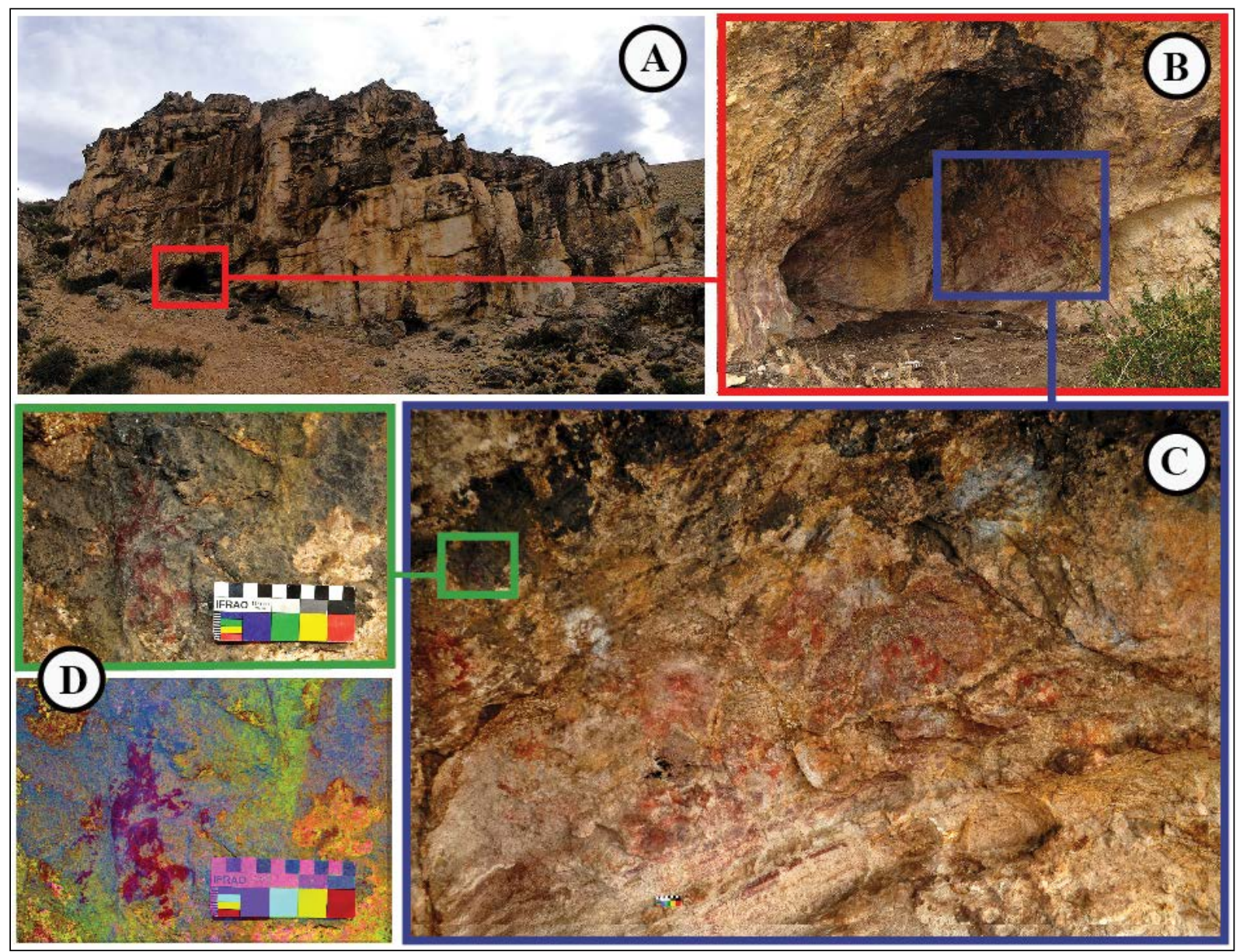

Figura 2. Alero Almendra: $A$ y B) vista general y particular del alero; C) panel con manos en color rojo, ocre y blanco; D) figura zoomorfa: arriba original, abajo tratada con DStretch.

\section{Materiales y métodos}

Sobre la base de un relevamiento bibliográfico previo, las tareas realizadas en el cañadón Caracoles incluyeron: 1) transectas dirigidas a la identificación de los sitios mencionados por Gradin et al. (1979), 2) recolecciones de superficie y 3) registro y geolocalización de nuevos conjuntos. Para las tareas de campo se utilizaron dos estrategias: a) prospecciones siguiendo el recorrido natural del cañadón y b) una vez identificados sectores con concentración de material, se realizaron una serie de transectas radiales con el fin determinar los tamaños máximos de dispersión de las concentraciones. Todas estas actividades fueron desarrolladas por ocho operadores que tenían una distancia de 20 m entre sí. Se recolectó el material en las concentraciones identificadas y, para evaluar la potencia estratigráfica se realizaron sondeos exploratorios de $0,50 \mathrm{~m}$ por $0,50 \mathrm{~m}$ decapando a través de niveles de depositación naturales. En el caso de los sitios bajo reparo que pudieron identificarse, no se realizaron recolecciones de superficie, sino que se realizaron relevamientos planimétricos expeditivos para, en futuras campañas, realizar los trabajos intensivos correspondientes.

El análisis de los conjuntos líticos se realizó siguiendo la propuesta de Aschero (1975, 1983) y Aschero y Hocsman (2004). Los materiales fueron separados de acuerdo a su origen y divididos por materias primas, clases tipológicas y, para el caso de los artefactos formatizados, grupos tipológicos. 
En el caso del arte rupestre, en campo se realizó un registro fotográfico de los paneles con manifestaciones, un recuento preliminar de los motivos presentes y un croquis a mano alzada del sitio en el que se identificaron. Luego se procesaron en gabinete las fotografías con los programas Photoshop y DStretch para obtener un primer acercamiento al registro de arte rupestre del sitio.

\section{Resultados}

En la parte baja del cañadón se registró un total de tres concentraciones superficiales en las que se procedió a recolectar el material (ver Tabla 1 y Figura 1B). En la primera de ellas, Confluencia I, se recuperó la mayor cantidad de artefactos de las tres concentraciones, incluyendo: 13 fragmentos cerámicos (tiestos) junto con cuatro artefactos líticos planos que podrían ser fragmentos de artefactos de molienda. En otra de las concentraciones, denominada Confluencia III, apareció una cuenta de collar y en Confluencia VI, fragmentos de vidrio $(n=25)$.

En el caso del registro lítico, como puede observarse en las Tablas 1 y 2, se analizó el material de ambas concentraciones por separado. En el caso de Confluencia I (Tabla 2), predominan las rocas silíceas (70\%) por sobre la obsidiana (19\%), ambas materias primas de muy buena calidad para la talla. En el caso de las rocas silíceas se encuentran disponibles en las cercanías, como por ejemplo el caso de la cantera Bosque Grande (a unos 50 km aproximadamente), donde Mengoni Goñalons (1987) informa la presencia de rocas silíceas de buena calidad que se presentan en nódulos tabulares. Mientras que la obsidiana está a unos 100 km lineales (Belardi et al., 2006; Espinosa y Goñi, 1999). Por otro lado, desde el punto de vista tecnológico, en las clases tipológicas representadas se observa un predominio de los desechos de talla (91\%; frecuencia respecto al total de artefactos líticos $n=1.968 / 2.145)$, seguido por los artefactos formatizados (6,5\%; ídem anterior $n=141 / 2.145)$ y los núcleos $(1,2 \%$; $n=26 / 2.145$ ). Por último, debemos destacar que los grupos tipológicos (Tabla 2) más representados dentro de los artefactos formatizados fueron los fragmentos de filo $(35,4 \%)$, seguido por las puntas de proyectil $(30,4 \%)$ y los raspadores $(23,4 \%)$.

\begin{tabular}{|c|c|c|c|c|c|c|c|c|c|c|}
\hline \multicolumn{6}{|c|}{ Confluencia I } & \multicolumn{5}{|c|}{ Confluencia III } \\
\hline \multicolumn{2}{|c|}{ GT/MP } & SI & OB & Otras & Total & GT/MP & SI & OB & Otras & Total \\
\hline \multirow{10}{*}{ 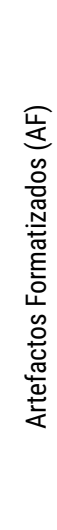 } & Raspador & 28 & 5 & - & 33 & Raspador & - & - & - & - \\
\hline & P. Proy & 31 & 12 & - & 43 & P. Proy & 2 & - & - & 2 \\
\hline & Perforador & 1 & - & - & 1 & Perforador & - & - & - & - \\
\hline & Frag. Filo & 43 & 2 & 5 & 50 & Frag. Filo & - & 2 & - & 2 \\
\hline & Raedera & - & 1 & 3 & 4 & Raedera & - & - & - & - \\
\hline & Art. Plano & - & - & 6 & 6 & Art. Plano & - & - & - & - \\
\hline & Muesca & - & - & 1 & 1 & Muesca & - & - & - & - \\
\hline & Percutores & - & - & 2 & 2 & Percutores & - & - & - & - \\
\hline & P. Destacada & 1 & - & - & 1 & P. Destacada & - & - & - & - \\
\hline & Total AF & 104 & 20 & 17 & 141 & Total AF & 2 & 2 & - & 4 \\
\hline \multicolumn{2}{|c|}{ Nucleos } & 13 & 13 & - & 26 & Nucleos & 1 & 3 & - & 4 \\
\hline \multicolumn{2}{|c|}{ FNRC } & 8 & 2 & - & 10 & FNRC & 4 & - & 2 & 6 \\
\hline \multicolumn{2}{|c|}{ Total } & 125 & 35 & 17 & 177 & Total & 7 & 5 & 2 & 14 \\
\hline
\end{tabular}

Tabla 2. Totales por grupo tipológico y clase tipológica del total de las recolecciones. Referencias: GT, grupo tipológico; MP, materia prima; SI, rocas silíceas; Ob, obsidiana; P. Proy, puntas de proyectil; Frag. Filo, fragmento de filo; P. Destacada, punta destacada; Art. Plano, artefacto plano, Total AF, total artefactos formatizados; FNRC, filos naturales con rastros complementarios. 
Entre los cabezales líticos se destacan las puntas pedunculadas pequeñas, del tipo Bird IV confeccionadas en rocas silíceas $(72,1 \%)$ y obsidiana (27,9\%). Como puede observarse en la Tabla 2, el $91 \%(n=43)$ de estas y el $96 \%(n=50)$ de los fragmentos de filo se encuentran en Confluencia I. Otro aspecto que llama la atención es la proporción de núcleos presentes. En la Tabla 2 puede observarse que un alto porcentaje de estos -en el caso de Confluencia III el 75\% ( $n=3)$ y $50 \%(n=13)$ en Confluencia I- se encuentran confeccionados en obsidiana a diferencia de lo que sucede con las puntas de proyectil.

Los fragmentos cerámicos recuperados no presentan ningún rasgo diagnóstico: presencia de puntos de inflexión o intersección, bordes o decoración (Balfet, Fauvet Berthelot y Monzón, 1983; Orton, Tyers y Vince, 1997) por lo que no puede hacerse una estimación acerca de las partes representadas o los $n$ totales. Se trata de fragmentos de pasta porosa de color gris posiblemente de cocción reductora.

Como se mencionó en la sección de materiales y métodos, se realizaron dos sondeos de $0,50 \mathrm{~m}$ por 0,50 $\mathrm{m}$ en la concentración denominada Confluencia I (Figura 1B). El objetivo de estos sondeos fue dar un anclaje temporal a los sitios, aunque ambos tuvieron como resultado pocas evidencias en estratigrafía. Entre estas se destacan algunas cáscaras de huevo, restos óseos en diferentes estados de conservación y algunos artefactos líticos (Tablas 1 y 2). Sin embargo, la poca integridad de los materiales orgánicos no permitió la toma de muestras para la realización de fechados radiocarbónicos.

Como se mencionó más arriba, a lo largo del recorrido del Caracoles existen varios cañadones secundarios que desembocan en él. En uno de ellos en particular, llamado cañadón Almendra, se identificaron cuatro aleros (ver Figura 1C) entre los que se destaca el Alero Almendra mencionado por Gradin et al. (1979). Este pequeño alero (4,50 $\mathrm{m}$ de boca, 3,10 $\mathrm{m}$ de altura y $4,90 \mathrm{~m}$ de profundidad) se encuentra sobre un paredón de aproximadamente $16 \mathrm{~m}$ de largo con orientación Noreste, presenta paneles con motivos rupestres (Figura $2 \mathrm{~A}$ y $\mathrm{B}$ ) y algunas lascas en superficie. La mayor concentración de motivos se encuentra al interior de este sector, que ofrece gran reparo. Las representaciones se encontraron sumamente desvaídas y en algunos casos afectadas por los procesos de exfoliación de la roca soporte. Por esta razón, fue necesaria la utilización de DStretch (Harman, 2008) en gabinete para analizar las fotografías tomadas en campo y conseguir una mejor apreciación del arte del sitio. Allí se registraron hasta el momento 26 negativos de mano correspondientes a adultos, en tonalidades ocre-amarrillo, rojo y blanco y uno de menor tamaño, probablemente de infante, en blanco. Debido al grado de deterioro no fue posible identificar en todos los casos si se trataba de manos derechas o izquierdas, pero las 18 que sí pudieron ser claramente definidas, se trataba de manos izquierdas. La principal concentración de motivos se encuentra distribuida aproximadamente entre los 50 y $200 \mathrm{~cm}$ sobre el piso actual del alero (Figura 2C). Además de los negativos de mano ya registrados en las menciones previas del sitio (Gradin et al. 1976, 1979), en la revisión se pudo identificar una figura zoomorfa en rojo (Figura 2D). Al exterior del alero se observaron al menos otros cinco negativos de mano en rojo a lo largo de cuatro unidades topográficas. Sin embargo, queda pendiente llevar a cabo un relevamiento exhaustivo de las representaciones rupestres del sitio. La revisión de las imágenes en gabinete expuso una mayor concentración de motivos que la identificada en campo y las imágenes tomadas son insuficientes para establecer un buen registro del arte del Alero Almendra.

Ubicado unos 100 m cañadón arriba del Alero Almendra se encuentra el sitio Almendra Histórico (Figura 1C). Se trata de un alero de dimensiones pequeñas que se encuentra acondicionado con una pirca de unos $50 \mathrm{~cm}$ de alto y en sus paredes internas puede observarse un espacio utilizado como fogón (evidenciado por el tiznado de la pared adyacente) con una serie de alambres asociados al posible colgado de recipientes para calentar agua. Asimismo, se identificaron herraduras utilizadas como ganchos en las paredes, desechos de material de origen industrial (vidrios, gres y metales) y material lítico en el talud. Se destaca que tanto este sitio, como el descripto 
anteriormente presentan sedimentación que hace suponer la existencia de potencial estratigráfico de interés arqueológico (Figura 3A, B y C).

Cañadón abajo, llegando a la confluencia del Almendra con el Caracoles, se encuentra el Alero del Puesto (ver Figura 1C). Se trata del aprovechamiento de una saliente de la roca a la que, con material vegetal local (probablemente mata negra) se acondicionó el espacio, generando un vano y paredes que segmentan el lugar (Figura 3D). Al interior se pudieron observar, entre otros materiales históricos, latas $(n=6)$, frascos $(n=3)$, pavas $(n=2)$, un plato enlozado, un banco confeccionado sobre material vegetal local y ganchos realizados con alambres en las paredes. De acuerdo con los datos aportados por los pobladores locales, este espacio era utilizado hasta épocas recientes por los puesteros de la zona.

Finalmente, sobre la margen izquierda del cañadón Caracoles, frente a la desembocadura del Almendra se encuentra el Alero Confluencia VIII (ver Figura 1C). Es un abrigo amplio que además presenta una pared pircada de unos $70 \mathrm{~cm}$ de altura, pero sin material en superficie ni evidencias de manifestaciones rupestres. Aunque tampoco se observaron rastros de tiznado en las paredes adyacentes al sector pircado, la presencia de sedimento acumulado hace que sea uno de los potenciales sitios a ser sondeados. La mayoría de los aleros relevados corresponden a ocupaciones subactuales con presencia de material de origen industrial como clavos (redondos y algunos con cabeza cuadrada), metales y herraduras. Además, como en el caso de los aleros denominados Almendra Histórico y Alero Confluencia VIII, se destaca la estructuración del espacio por medio del pircado. Por otro lado, en el Alero del Puesto esta estructuración se logró a partir de la construcción de paredes con especies vegetales locales (Figura 3D).
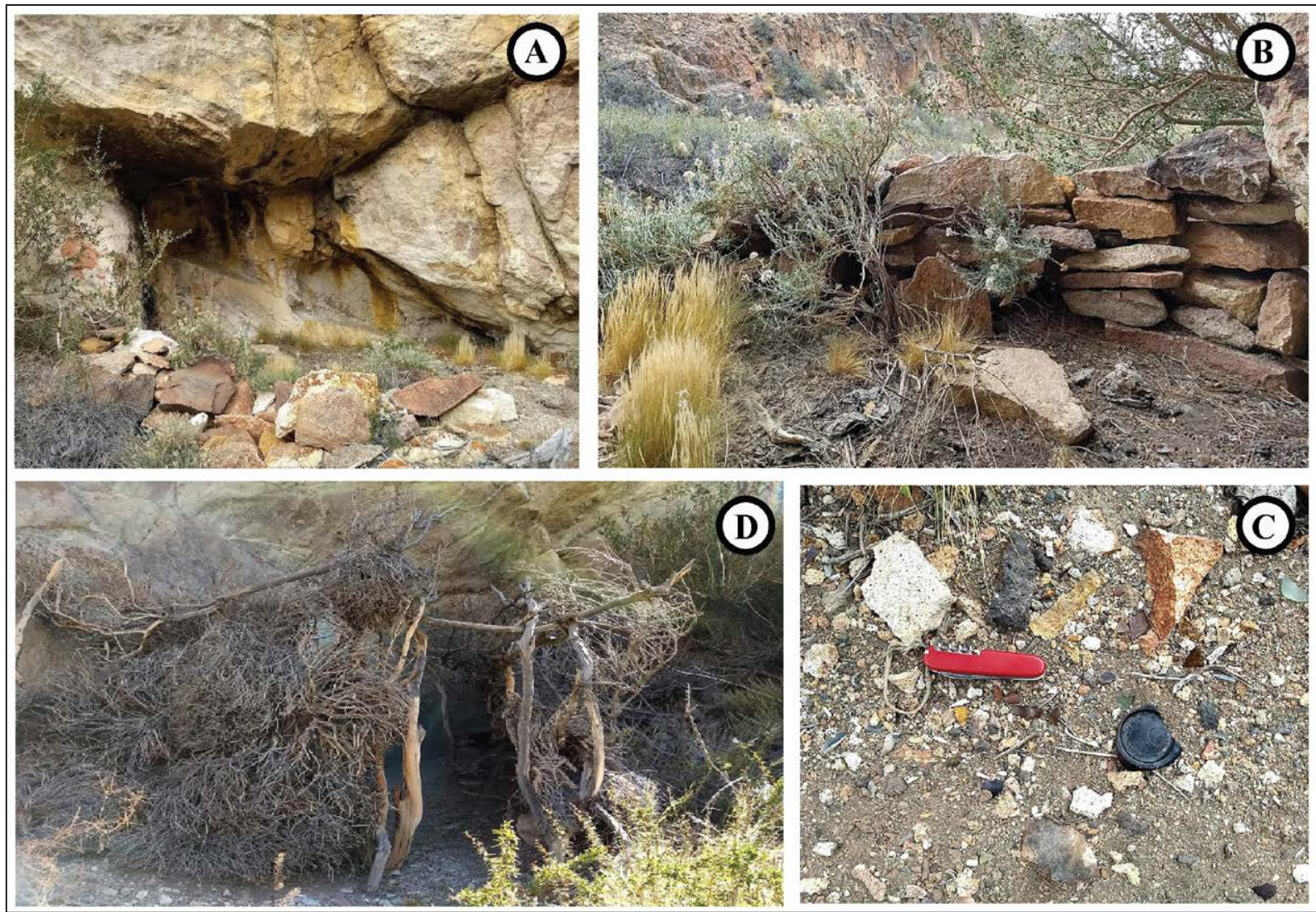

Figura 3. A) vista general de Almendra Histórico; B) detalle del pircado; C) detalle de materiales en talud; D) vista del Alero del Puesto. 


\section{Discusión y consideraciones finales}

Como se mencionó en la introducción, este trabajo se enmarca en proyectos que buscan evaluar e identificar posibles vías de comunicación entre las zonas lacustres cordilleranas y la zona de estepa hacia el este. Una de estas potenciales vías de circulación desde el río Pinturas hacia el oeste sería el recorrido del cañadón Caracoles hacia el sector lacustre cordillerano. En este sentido, uno de los objetivos de las prospecciones y sondeos se orientó a la identificación de la profundidad temporal en el uso de este espacio. Sin embargo, quedó inconcluso al no recuperarse material datable en los sondeos realizados. Es así que queda pendiente la realización de nuevas excavaciones que puedan dar como resultado material orgánico en estratigrafía para obtener una fecha mínima de inicio en el uso de este espacio, estratégico a partir de lo registrado en la parte baja del cañadón. Por un lado, la cercanía de Cueva de las Manos y Arroyo Feo, que presentan fechados que rondan los ca. 10.700 años cal. AP (Aschero et al., 2019b), así como los fechados de Cerro Casa de Piedra 7 en el PNPM de ca. 12.500 años cal. AP (Aschero et al. 2019a) y Cueva del Milodon Norte 1 y Cerro Cuadrado 3 en el lago Pueyrredón de ca. 8500 años cal. AP (Aschero et al., 2019a), hace pensar acerca del rol que pudo haber ocupado el cañadón Caracoles en la circulación desde la estepa hacia los espacios cordilleranos tanto para los grupos cazadores como para las tropillas de guanacos en sus movimientos estacionales. En este sentido, las excavaciones en sitios como Alero Almendra junto con la prospección y ubicación de nuevos sitios a lo largo del cañadón y de sus subsidiarios nos permitirán discutir el uso de estos espacios a lo largo del Holoceno. Por otro lado, las características de los materiales recuperados en superficie, ubicados en la parte baja del cañadón Caracoles, coinciden con lo expuesto por Gradin et al. (1979). A partir de las recolecciones que realizaron, estos autores plantean que los sitios ubicados en la zona de la confluencia entre el cañadón Caracoles y el río Pinturas habrían sido ocupados durante el Holoceno tardío. Sin embargo, por lo expuesto más arriba consideramos posible el uso de estos espacios desde momentos más tempranos, aspecto que deberá ser evaluado en mayor profundidad en el futuro.

Las cronologías para los momentos más recientes disponibles en áreas cercanas como el PNPM y los lagos Salitroso y Posadas varían entre $960 \pm 125$ y $240 \pm 50$ años AP en contextos con materiales cerámicos (Cassiodoro y Tessone, 2014). Gradin y Aguerre (1991), en consonancia con lo anterior, indican que contextos similares en el área del río Pinturas tienen una cronología de ca. 1100 años AP. La evidencia lítica que acompaña a estos materiales también parece adecuarse a este planteo dada la presencia de artefactos planos (posibles manos y molinos), puntas pedunculadas pequeñas y artefactos de módulo pequeño confeccionados sobre hojas. Queda pendiente la realización de análisis de microdesgaste y de residuos sobre los posibles artefactos de molienda con el objetivo de identificar si su uso se asocia con minerales o vegetales. Asimismo, otra de las tareas pendientes es la identificación de las fuentes de obtención de las rocas silíceas. Por un lado, se cuenta con la fuente potencial identificada por Mengoni Goñalons (1987) antes mencionada. A esto debe sumarse que en los últmos años se identificaron dos posibles canteras de este tipo de materia prima. Se trata, en primer lugar, de un afloramiento localizado en las proximidades de la laguna Cisnes, ubicada a alrededor de $40 \mathrm{~km}$ hacia el sur de la confluencia entre el cañadón Caracoles y el río Pinturas. Por otro lado, cerca de la entrada vehicular desde la ruta nacional 40 hacia la Cueva Grande de Arroyo Feo se encuentra el cerrillo del Puma, a unos $15 \mathrm{~km}$ al norte de la confluencia antes mencionada. Estas localidades aún deben ser analizadas y muestreadas.

Con respecto al Alero Almendra, en las publicaciones originales Gradin et al. (1976, 1979) hacen referencia únicamente a los motivos de negativos de mano, por lo que el hallazgo de una figura zoomorfa de color rojo en el contexto de estos relevamientos es algo a destacar. Es por esta razón que dentro de las tareas a realizar en las futuras campañas se incluirá el relevamiento exhaustivo y sistemático de los paneles de este sitio. En cuanto al Alero Almendra Histórico, ubicado a $100 \mathrm{~m}$ del anterior, la presencia de material de origen local e industrial en superficie y en el talud podría ser 
un indicador de continuidad o reutilización de los espacios a lo largo del tiempo (Goñi 2000, 2000-2002a, 2000-2002b, 2010; Goñi, Belardi, Espinosa y Savanti, 2004; Nuevo Delaunay, 2012, 2015; Nuevo Delaunay et al., 2017; Sacchi y Saletta, 2017; Saletta, 2015; Saletta y Sacchi, 2019; entre otros). En este sentido, los datos aportados por Aguerre (1990-1992, 2000) y Priegue (2007) acerca de los lugares elegidos por las familias tehuelches que se localizaron en el río Pinturas a principios del siglo XX indicarían un conocimiento previo del espacio y el paisaje que apoyaría esta interpretación. Incluso algunos de estos sitios podrían vincularse con los movimientos a los que hacen referencia las autoras. Estos territorios eran conocidos y ocupados en distintos momentos del año (Aschero et al., 2019b; Gradin et al., 1976, 1979; entre otros). Sin embargo, como plantean Goñi y Nuevo Delaunay (2009), producto de los avances del Estado Nación a fines del siglo XIX, los grupos tuvieron que modificar sus estrategias de movilidad. Estas nuevas estrategias habrían estado caracterizadas por el uso de espacios marginales a la ganadería lanera. De esta manera, se buscó minimizar el contacto con los agentes estatales, por un lado, pero también con los ganaderos, por el otro (Nuevo Delaunay, 2012, 2015). Estas fueron respuestas estratégicas frente al avance de los establecimientos ganaderos y las reservas indígenas. De acuerdo a la información relevada por Aguerre (1990-1992, 2000), hubo grupos que utilizaron el área del río Pinturas hasta la década de 1940 y luego se siguió utilizando como parte del circuito productivo como espacios usados por los puesteros para descansar.

Finalmente, estos relevamientos evidenciaron la necesidad de realizar una serie de prospecciones sistemáticas del total del cañadón. De esta manera se incluiría el sector inicial del cañadón, hacia la zona de Bajo Caracoles y la actual ruta nacional 40. Los relevamientos de superficie se suman a la excavación de sitios en estratigrafía, ubicados en los aleros de los cañadones secundarios que tributan al Caracoles. El desarrollo de estas actividades permitirá avanzar en el establecimiento de una cronología para el inicio del uso de este espacio. De esta manera será posible discutir su momento de ingreso como alternativa para la circulación entre la cordillera y la estepa.

\section{Agradecimientos}

Este trabajo es parte de distintos proyectos financiados por la Universidad de Buenos Aires (UBACyT 20020170200085BA) y la Agencia Nacional de Promoción Científica y Tecnológica (PICT 2013-2240). Queremos agradecer especialmente a la Municipalidad de Perito Moreno por el apoyo recibido, a la Asociación Identidad, al Museo de Arqueología Carlos J. Gradin, a Carolina Ávila, Ricardo Vázquez, a los propietarios de la Estancia Casa de Piedra, Héctor y Cinecia Sabella, a la Fundación Flora y Fauna Argentina propietaria de la Estancia Los Toldos. Al equipo de trabajo que nos acompañó en la campaña 2018, Marcos Rambla y Carlos Masotta. Queremos agradecer especialmente a Teresa Civalero por la lectura de una de las primeras versiones del manuscrito y por siempre empujarnos a hacer algo más. A María José Saletta, por sus comentarios y la ayuda bibliográfica sobre momentos históricos. A Carlos Aschero y Anette Aguerre por los comentarios, los datos sobre el cañadón, por la ayuda, por brindarnos siempre una mano y compartir sus experiencias con nosotros. Finalmente queremos agradecer a los dos evaluadores anónimos cuyas críticas, observaciones y correcciones mejoraron notablemente este trabajo. Lo vertido aquí es de nuestra exclusiva responsabilidad. 


\section{Referencias citadas}

» Aguerre, A. M. (1990-1992). Familias aborígenes del área del Río Pinturas, Noroeste de la provincia de Santa Cruz. Relaciones de la Sociedad Argentina de Antropología, XVIII, 139-180.

» Aguerre, A. M. (2000). Las Vidas de Pati en la toldería Tehuelche del Río Pinturas y el después: Provincia de Santa Cruz, Argentina. Buenos Aires: Universidad de Buenos Aires, Facultad de Filosofía y Letras.

»Aguerre, A. M. (Ed). (2003). Arqueología y Paleoambiente en la Patagonia Santacruceña Argentina. Buenos Aires: Talleres Gráficos Nuevo Offset.

» Aguerre, A. M. y Gradin, C. J. (2003). La dinámica poblacional del curso medio del Río Pinturas. En A. M Aguerre (Ed.), Arqueología y paleoambiente en la Patagonia santacruceña argentina (pp. 153-204). Buenos Aires: Talleres Gráficos Nuevo Offset.

» Alonso, F., Gradin, C. J., Aschero, C. A. y Aguerre A. M. (1984-1985). Algunas consideraciones sobre recientes dataciones radiocarbónicas para el Río Pinturas, Provincia de Santa Cruz. Relaciones de la Sociedad Argentina de Antropología, XVI, 275-285.

»Aschero, C. A. (1975). Ensayo para una clasificación morfológica de artefactos líticos aplicada a estudios tipológicos comparativos. Informe al CONICET. Manuscrito inédito.

»Aschero, C. A. (1981-1982). Nuevos datos sobre la Arqueología del Cerro Casa de Piedra. Relaciones de la Sociedad Argentina de Antropología, XIV(2), 267-284.

» Aschero, C. A. (1983). Ensayo para una clasificación morfológica de artefactos líticos aplicada a estudios tipológicos comparativos. Apéndices A-C. Revisión. Buenos Aires: Cátedra de Ergología y Tecnología (FFyL, UBA). Manuscrito inédito.

» Aschero, C. A., Bozzuto, D. L., Civalero, M. T., De Nigris, M. E., Di Vruno, A., Dolce, V., Fernández, N. L., González, L. y Limbrunner, P. (2009). El registro arqueológico de la costa noreste del Lago Pueyrredón-Cochrane (Santa Cruz, Argentina). En M. Salemme, F. Santiago, M. Álvarez, E. Piana, M. Vázquez y E. Mansur (Eds.), Arqueología de la Patagonia. Una mirada desde el último confín (Tomo 2, pp. 919-926). Ushuaia: Utopías.

» Aschero, C. A., Bozzuto, D. L., Civalero, M. T., De Nigris, M. E., Fernández, N. L., Maveroff, N. y Sacchi, M. (2019a). Se nos viene la noche. El volcán Hudson y su influencia en el NO de Santa Cruz: integrando perspectivas arqueológicas. En J. Gómez Otero, A. Svoboda y A. Banegas (Eds.), Arqueología de la Patagonia: el pasado en las arenas (pp. 239-249). Puerto Madryn: CONICET, Instituto de Diversidad y Evolución Austral.

"Aschero, C. A. y Hocsman, S. (2004). Revisando cuestiones tipológicas en torno a la clasificación de artefactos bifaciales. En M. Ramos, A. Acosta y D. Loponte (Comps.), Temas de Arqueología. Análisis Lítico (pp. 7-25). Luján: Universidad Nacional de Luján.

» Aschero, C. A., Isasmendi, M. V., Ucedo, V. R. y Aguerre, A. M. (2019b). Aportes a la cronología y contexto de las escenas de caza tempranas en Cueva de las Manos (ca. 9400-7700 años AP). En J. Gómez Otero, A. Svoboda y A. Banegas (Eds.), Arqueología de la Patagonia: el pasado en las arenas (pp. 41-51). Puerto Madryn: Instituto de Diversidad y Evolución Austral.

» Balfet, H., Fauvet Berthelot, M. H. y Monzón, S. (1983). Pour la Normalisation de la Déscription des Poteries. Paris: Centre National de la Recherche Scientifique.

» Belardi, J. B. (2004). Más vueltas que una greca. En M.T. Civalero, P. Fernández y G. Guraieb (Eds.), Contra viento y marea. Arqueología de Patagonia (pp. 591-603). Buenos Aires: Instituto Nacional de Antropología y Pensamiento Latinoamericano - Sociedad Argentina de Antropología.

» Belardi, J. B., Tiberi, P., Stern, C. R. y Súnico, A. (2006). Al este del Cerro Pampa: ampliación del área de disponibilidad de obsidiana de la Pampa del Asador (Provincia de Santa Cruz). Intersecciones en Antropología, 7, 27-36. 
"Cassiodoro, G. y Tessone, A. (2014). Análisis radiocarbónico y de isótopos estables en residuos cerámicos del centro-oeste de Santa Cruz (Patagonia). Relaciones de la Sociedad Argentina de Antropología, XXXIX(1), 293-299.

» Civalero, M. T. (2016). Propuesta metodológica para el análisis del material lítico del sitio Playa Cisnes 2, provincia de Santa Cruz, Patagonia argentina. En F. Mena (Ed.), Arqueología de Patagonia: de mar a mar (pp. 235-244). Coyhaique: Ñire Negro-CIEP.

» Civalero, M. T. y Aschero, C. A. (2003). Early Occupations at Cerro Casa de Piedra 7, Santa Cruz Province. Patagonia Argentina. En L. Miotti, M. Salemme y N. Flegenheimer (Eds.), Where the South Winds Blow: Ancient Evidences for Paleo South Americans (pp. 141-147). Texas: A\&M University Press.

» Decreto Nacional 817/2019. Declaración de Monumentos, Lugares y Bienes Históricos ubicados en la Provincia de Santa Cruz. Promulgado por el Poder Ejecutivo Nacional en Buenos Aires el 05 de diciembre de 2019. Boletín Oficial, 06 de diciembre de 2019.

»Escalada, F. (1949). El complejo 'tehuelche'. Estudios de etnografía patagónica. Buenos Aires: Coni.

» Espinosa, S. y Goñi, R. (1999). Viven: una fuente de obsidiana en la provincia de Santa Cruz. En Soplando en el Viento. Actas de las Terceras Jornadas de Arqueología de la Patagonia (Volumen 1, pp. 177-188). Neuquén-Buenos Aires: Universidad Nacional del Comahue e Instituto Nacional de Antropología y Pensamiento Latinoamericano.

»Fernández, N. L., Bozzuto, D. L., Sacchi, M., Maveroff, N., Civalero, M. T. y Aschero, C. A. (2019). Excavaciones en el alero Cerro Bayo 2, estepa oriental de Patagonia centro meridional. En T. Costa da Silva (Ed.), Libro de Resúmenes XX Congreso Nacional de Arqueología Argentina: 50 años de arqueologías (pp. 285-286). Córdoba: Facultad de Filosofía y Humanidades, Universidad Nacional de Córdoba.

» Geuna, S. E. y Escosteguy, L. D. (2008). El valle del río Pinturas. La Cueva de las Manos. Sitios de Interés Geológico de la República Argentina, 46(2), 771-780.

"Goñi, R. (2000). Arqueología de momentos históricos fuera de los centros de conquista y colonización: un análisis de caso en el sur de la Patagonia. En J. B. Belardi, F. Carballo Marina y S. Espinosa (Eds.), Desde el País de los Gigantes. Perspectivas Arqueológicas en Patagonia (pp. 283-296). Río Gallegos: Universidad Nacional de la Patagonia Austral.

»Goñi, R. (2000-2002a). Poblamiento humano, paleoambientes y cronología en la cuenca de los lagos Cardiel y Strobel. Cuadernos del Instituto Nacional de Antropología y Pensamiento Latinoamericano, 19, 669-671.

"Goñi, R. (2000-2002b). Fechados radiocarbónicos y registro arqueológico en la cuenca de los lagos Salitroso/Posadas (Santa Cruz). Cuadernos del Instituto Nacional de Antropología y Pensamiento Latinoamericano, 19, 666-668.

"Goñi, R. (2010). Cambio climático y poblamiento humano durante el Holoceno tardío en Patagonia Meridional. Una perspectiva arqueológica. (Tesis Doctoral inédita), Universidad de Buenos Aires, Argentina.

" Goñi, R. A., Belardi, J. B., Espinosa, S. L. y Savanti, F. (2004). Más vale tarde que nunca cronología de las ocupaciones cazadoras-recolectoras en la cuenca del lago Cardiel (Santa Cruz, Argentina). En M. T. Civalero, P. Fernández y G. Guraieb (Eds.), Contra viento y marea. Arqueología de Patagonia (pp. 237-248). Buenos Aires: Instituto Nacional de Antropología y Pensamiento Latinoamericano - Sociedad Argentina de Antropología.

» Goñi, R. A. y Nuevo Delaunay, A. (2009). La Arqueología como "fuente" de la Historia. En M. Salemme, F. Santiago, M. Álvarez, E. Piana, M. Vázquez y E. Mansur (Eds.), Arqueología de la Patagonia. Una mirada desde el último confín (pp. 149-158). Ushuaia: Utopías.

"Gradin, C. J. y Aguerre, A. M. (1988). Algunos aspectos de la dinámica poblacional en la cuenca del Río Pinturas. En Precirculados del IX Congreso Nacional de Arqueología Argentina (pp. 152-163). Buenos Aires: Universidad de Buenos Aires.

» Gradin, C. J. y Aguerre, A. M. (1991). Ocupaciones Patagonienses de la Capa 3 del Alero Cárdenas (Área Río Pinturas), Provincia de Santa Cruz. Arqueología, 1, 197-205. 
» Gradin, C. J., Aschero, C. A. y Aguerre, A. M. (1976). Investigaciones Arqueológicas en la Cueva de las Manos, Estancia Alto Río Pinturas, Pcia. de Santa Cruz. Relaciones de la Sociedad Argentina de Antropología, X, 201-270.

» Gradin, C. J., Aschero C. A. y Aguerre, A. M. (1979). Arqueología del Área Río Pinturas (Santa Cruz). Relaciones de la Sociedad Argentina de Antropología, XIII,183-227.

» Gradin, C. J., Aschero, C. A. y Aguerre, A. M. (1987). Primeros niveles culturales en el área Rio Pinturas (Provincia de Santa Cruz, Argentina). Estudios Atacameños, 8, 118-141.

» Harman, J. (2008). Using Decorrelation Stretch to Enhance Rock Art Images. http://www. dstretch.com/AlgorithmDescription.html. (Acceso: 20 de agosto, 2016).

» Horta, L. R., Marcos, M. A, Bozzuto, D. L., Mancini, M. V. y Sacchi, M. (2016). Paleogeographic and paleoenvironmental variations of Pueyrredón Lake - Posadas - Salitroso area during the Holoceneand their relationship to occupational dynamics. Palaeogeography, Palaeoclimatology, Palaeoecology, 449, 541-552. https://doi.org/10.1016/j.palaeo.2016.02.051

» Horta, L.R., Marcos, M.A., Sacchi, M., Bozzuto, D.L., Civalero, M.T., Georgieff, S. y Mancini, M.V. (2019). Paleogeographic and paleoenvironmental evolution in northwestern Santa Cruz (Argentina), and its influence in human occupational dynamics during the late Pleistoceneearly Holocene. Palaeogeography, Palaeoclimatology, Palaeoecology, 5(16), 44-53. https://doi. org/10.1016/j.palaeo.2018.11.037

» Ley Provincial 3.613/2018. Crea el "Parque Provincial Cueva de las Manos". Promulgada en Río Gallegos el 13 de septiembre de 2018. Boletín Oficial Provincia de Santa Cruz, 4 de octubre de 2018.

» Ley Provincial 3.394/2014. Declara paisaje natural y cultural "cuenca media e inferior del río Pinturas". Promulgada en Río Gallegos el 25 de septiembre de 2014. Boletín Oficial provincia de Santa Cruz, 27 de noviembre de 2014. Id SAIJ: LPZ0003394.

» Mengoni Goñalons, G. (1987). Investigaciones Arqueológicas en el noroeste de la meseta central de Santa Cruz. En Primeras Jornadas de Arqueología de la Patagonia. Serie Humanidades 2 (pp. 171-175). Rawson: Gobierno de la provincia de Chubut.

» Musters, G. C. (1911). Vida entre los patagones. Buenos Aires: Coni.

» Nuevo Delaunay, A. (2012). Disarticulation of Aónikenk hunter-gatherer lifeways during the late nineteenth and early twentieth centuries: two case studies from Argentinean Patagonia. Historical Archaeology, 46, 149-64. https://doi.org/10.1007/BF03376875

» Nuevo Delaunay, A. (2015). Transformación del paisaje arqueológico rural en el centro-oeste de la provincia de Santa Cruz, siglo XX. (Tesis Doctoral inédita), Universidad de Buenos Aires, Argentina.

» Nuevo Delaunay, A., Belardi, J. B., Carballo Marina, F., Saletta, M. J y De Angelis, H. (2017). Glass and stoneware knapped tools among hunter-gatherers in southern Patagonia and Tierra del Fuego. Antiquity, 91(359), 1330-1343. https://doi.org/10.15184/aqy.2017.125

» Orton, C., Tyers, P. y Vince, A. (1997). La cerámica en arqueología. Barcelona: Crítica.

»Priegue, C. N. (2007). En Memoria de los Abuelos. Historia de Vida de Luisa Pascual, Tehuelche. Bahía Blanca: Publitek.

» Sacchi, M., Bozzuto, D. L., Horta, L. R., Fernández, N. L., De Nigris, M. E., Civalero, M. T. y Aschero, C. A. (2016). Dataciones y circulación humana: Posibles influencias de las fluctuaciones del sistema lacustre Pueyrredón Posadas durante el holoceno. Andes, 27(2). http://portalderevistas. unsa.edu.ar/ojs/index.php/Andes/article/view/223/210

» Sacchi, M. y Saletta, M. J. (2017). Des-armando el tardío: variaciones en puntas de proyectil, boleadoras, raspadores, raederas y sus materias primas en momentos pre y postcontacto en Patagonia meridional. Ponencia presentada en las $\mathrm{X}$ Jornadas de Arqueología de la Patagonia, Puerto Madryn, Argentina.

»Saletta, M. J. (2015). Excavando fuentes: la tecnología, subsistencia, movilidad y los sistemas simbólicos de Shelk'nam, Yámana/Yaghan y Aonikenk entre los siglos XVI y XX analizados a partir de los registros escritos y arqueológicos. (Tesis Doctoral inédita), Universidad de Buenos Aires, Argentina. 
»Saletta, M. J. y Sacchi, M. (2019). ¿Ausencias o abandonos? Las puntas de proyectil en sitios postcontacto de Patagonia meridional (siglos XVI al XX). Revista de Estudios Sociales, 67, 101-114. 